\title{
Correlação entre a nasofibrofaringoscopia e a cefalometria no diagnóstico de hiperplasia de tonsilas faríngeas
}

\section{Correlation between nasopharyngoscopy and cephalometry in the diagnosis of hyperplasia of the pharyngeal tonsils}

\author{
Rodrigo Agne Ritzel', Luana Cristina Berwig', Ana Maria Toniolo da Silva ${ }^{3}$, Eliane Castilhos Rodrigues Corrêa4, \\ Eliane Oliveira Serpa ${ }^{5}$.
}

1) Mestre. Otorrinolaringologista do Hospital Universitário de Santa Maria.

2) Mestre. Fonoaudióloga Residente do Programa de Residência Multiprofissional Integrada em Gestão e Atenção Hospitalar no Sistema Público de Saúde da Universidade Federal de Santa Maria.

3) Doutora. Professora Associada do Curso de Fonoaudiologia da Universidade Federal de Santa Maria.

4) Doutora. Professora Adjunta do Curso de Fisioterapia da Universidade Federal de Santa Maria.

5) Mestre. Ortodontista clínica.

Instituição: Universidade Federal de Santa Maria. Santa Maria / RS - Brasil.

Endereço para correspondência: Rodrigo Agne Ritzel - Rua Pinheiro Machado 2350/806 - Bairro Centro - Santa Maria / RS - Brasil - CEP: 97050-600 - Telefone: (+55 55) 3028-9507 - E-mail: rodrigoritzel@ymail.com

Artigo recebido em 17 de Agosto de 2011. Artigo aprovado em 6 de Fevereiro de 2012.

\section{RESUMO}

Introdução: A hiperplasia de tonsila faríngea é uma das principais causas da respiração oral. O diagnóstico preciso desta alteração é importante para o correto planejamento terapêutico. Em vista disso, estudos têm sido desenvolvidos a fim de fornecer subsídios quanto aos procedimentos que podem ser utilizados para o diagnóstico de obstrução faríngea.

Objetivo: Verificar a correlação entre os exames de nasofibrofaringoscopia e cefalometria no diagnóstico de hiperplasia de tonsila faríngea.

Método: Estudo transversal, clínico e experimental. Participaram deste estudo 55 crianças, 30 meninas e 25 meninos, com idades entre 7 e 11 anos. As crianças foram submetidas à avaliação nasofibrofaringoscópica e cefalométrica para a determinação do grau de obstrução da nasofaringe. Para verificar a correlação entre esses exames foi utilizado o coeficiente de correlação de Spearman ao nível de significância de $5 \%$.

Resultados: Na nasofibrofaringoscopia a maioria das crianças apresentou hiperplasia de tonsila faríngea graus 2 e 3, seguidas de grau 1. Na cefalometria a maior parte das crianças apresentou hiperplasia de tonsilas faríngeas grau 1, seguida de grau 2. Na correlação entre os exames, evidenciou-se correlação regular e positiva.

Conclusão: A avaliação da hiperplasia de tonsilas faríngeas pode ser realizada pela nasofibrofaringoscopia e pela cefalometria, pois estes exames apresentam uma relação regular e positiva. No entanto, verificou-se que a cefalometria tende a subestimar o tamanho da tonsila faríngea em relação à nasofibrofaringoscopia.

Palavras-chave: respiração bucal, nasofaringe, diagnóstico, estudo comparativo.

\section{SUMMARY}

Introduction: Hyperplasia of the pharyngeal tonsil is one of the main causes of mouth breathing, and accurate diagnosis of this alteration is important for proper therapeutic planning. Therefore, studies have been conducted in order to provide information regarding the procedures that can be used for the diagnosis of pharyngeal obstruction.

Objective: To verify the correlation between nasopharyngoscopy and cephalometric examinations in the diagnosis of pharyngeal tonsil hyperplasia.

Method: This was a cross-sectional, clinical, experimental, and quantitative study. Fifty-five children took part in this study, 30 girls and 25 boys, aged between 7 and 11 years. The children underwent nasofibropharyngoscopic and cephalometric evaluation to determine the grade of nasopharyngeal obstruction. The Spearman's rank correlation coefficient at the $5 \%$ significance level was used to verify the correlation between these exams.

Results: In the nasopharyngoscopy evaluation, most children showed grade 2 and 3 hyperplasia of the pharyngeal tonsil, which was followed by grade 1 . In the cephalometry assessment, most children showed grade 1 hyperplasia of the pharyngeal tonsil, which was followed by grade 2 . A statistically significant regular positive correlation was observed between the exams.

Conclusion: It was concluded that the evaluation of the pharyngeal tonsil hyperplasia could be carried out by fiber optic nasopharyngoscopy and cephalometry, as these examinations were regularly correlated. However, it was found that cephalometry tended to underestimate the size of the pharyngeal tonsil relative to nasopharyngoscopy.

Keywords: mouth breathing, nasopharynx, diagnosis, comparative study. 


\section{INTRODUÇÃO}

O processo da respiração, que inicia nas vias aéreas superiores e culmina com a troca gasosa nos alvéolos, é vital para sobrevivência do ser humano. Quando a respiração inicia pelo nariz, o ar é preparado para chegar ao pulmão em condições ideais, ou seja, é aquecido, umidificado e filtrado, ativando processos imunológicos como transporte mucociliar e atividade microbicida que protegem a via aérea inferior. Quando a respiração inicia pela boca, apesar de haver aquecimento e umidificação do ar, essa não apresenta atividade de filtragem e imunológica (1).

A respiração oral quando presente na infância, fase de intenso crescimento muscular e esquelético da face, promove adaptação patológica das estruturas do sistema estomatognático em detrimento do harmonioso crescimento morfológico e funcional dessas estruturas (2).

As principais causas de respiração oral na infância são hipertrofia de tonsilas faríngea e/ou palatinas, edema de mucosa nasal e conchas nasais, desvio de septo, hábito de sucção por tempo prolongado, entre outras (3).

As adaptações morfológicas em crianças respiradoras orais ocorrem visando facilitar a necessária chegada do ar no alvéolo. Assim, pode-se observar hipoplasia maxilar e rebaixamento/rotação posterior da mandíbula, sendo que estas levam a alterações na oclusão dentária, maior inclinação mandibular e padrão de crescimento facial vertical, com alterações nas proporções faciais normais e elevação do palato duro, anteriorização da cabeça e desarmonia muscular principalmente da região orofacial. Essas adaptações geram desequilíbrio funcional do sistema estomatognático evidenciado por alterações na fala, mastigação e deglutição (4-6).

A complexidade das consequências da respiração oral associadas com as várias etiologias justifica a participação de diversos profissionais, como otorrinolaringologistas, odontólogos, fonoaudiólogos, fisioterapeutas, pediatras, entre outros, nas diversas fases do atendimento do respirador oral, tais como no diagnóstico, tratamento, reabilitação e na prevenção da respiração oral na infância. A integração da equipe multiprofissional é necessária, sendo desejável o uso de uma classificação uniforme dos respiradores orais, das mesmas terminologias e dos mesmos exames complementares.

O correto diagnóstico da causa de respiração oral é imprescindível para efetividade do tratamento. $\mathrm{Na}$ fonoaudiologia a conduta no atendimento de pacientes com respiração oral tem sido determinada pela etiologia do modo respiratório alterado. Portanto, o correto uso das opções diagnósticas colabora para o diagnóstico precoce, para auxiliarna definição de uma terapêutica multiprofissional mais adequada a cada caso e para minimizar as recidivas na reabilitação dos respiradores orais $(7,8)$.

A maioria das causas de respiração oralé diagnosticada pelo exame físico otorrinolaringológico desarmado, com exceção do diagnóstico de hiperplasia de tonsila faríngea, que necessita de exame complementar (3).

Para o diagnóstico de hiperplasia faríngea, os exames usualmente solicitados são radiografia de cavum e nasofibrofaringoscopia. Anasofibrofaringoscopia éum exame que visualiza a tonsila faríngea e sua relação com as demais estruturas da nasofaringe, sendo considerada por muitos autores como exame mais fidedigno para diagnóstico de obstrução da nasofaringe (9-13). A cefalometria é um exame semelhante à radiografia de cavum, entretanto realizado com uso de cefalostato, que possibilita o posicionamento mais adequado do paciente, fornece dados referentes ao de crescimento craniofacial e estado miofuncional destas estruturas(14). Por não ser um exame invasivo é mais confortável sua realização, sendo mais aceito especialmente na faixa etária das crianças, além de ter custo mais baixo que a nasofibrofaringoscopia e ser mais disponível.

Com a finalidade de contribuir com o diagnóstico preciso do respirador oral, realizou-se esta pesquisa que teve como objetivo verificar a correlação entre os exames de nasofibrofaringoscopia e cefalometria no diagnóstico de hiperplasia de tonsilas faríngeas.

\section{MÉTOdO}

Este estudo foi aprovado pelo Comitê de Ética em Pesquisa da instituição de origem sob o protocolo de número 220.0.243.000-8. As crianças assentiram a sua participação no estudo e tiveram o Termo de Consentimento Livre e Esclarecido assinado pelo responsável.

A amostra foi composta por crianças oriundas de três escolas da rede pública, sendo que as mesmas foram avaliadas entre setembro de 2008 e dezembro de 2009 . Os critérios de inclusão foram: apresentar respiração oral ou alguma queixa relacionada com respiração oral como baba noturna, ronco, sono agitado, alteração na oclusão dentária, hipotonia na musculatura orofacial, alteração de fala/ mastigação/deglutição; ter idade entre sete e 11 anos e 11 meses. Foram excluídas as crianças que apresentavam comprometimento neurológico evidente, má formação craniofacial, síndrome ou histórico de cirurgia faríngea. Não foi realizada diferenciação entre os sexos. 
Por meio dos critérios de inclusão e exclusão, foram selecionadas 55 crianças, 25 meninos e 30 meninas, com idade média de 9 anos e 8 meses. Essas crianças foram submetidas à avaliação otorrinolaringológica e aos exames de nasofibrofaringoscopia e cefalometria, através do quais foi verificada a existência ou não de obstrução na nasofaringe. Na amostra deste estudo não foram diagnosticadas outras causas de obstrução das vias aéreas superiores como desvio septal, hipertrofia primária de conchas nasais, pólipos e más formações. Para os pacientes com rinossinusite bacteriana e/ou rinite alérgica sintomática foram prescritos tratamentos e posterior reavaliação otorrinolaringológica em um período de 30 a 60 dias, sendo então realizados os exames de nasofibrofaringoscopia e cefalometria.

A avaliação otorrinolaringológica foi realizada na presença e com ajuda dos pais ou responsável, sendo que após anamnese geral e específica otorrinolaringológica com ênfase em aspecto relacionado com respiração oral/ nasal e demais funções orofaciais, foi realizado exame físico que constou de otoscopia, rinoscopia anterior, palpação cervical e oroscopia. Nesta avaliação era definido se o modo respiratório do paciente era oral ou nasal.
Imediatamente após a realização da anamnese e exame clínico otorrinolaringológico, foi realizado o exame de nasofibrofaringoscopia, sempre pelo mesmo avaliador e no mesmo local, com uso de anestesia tópica (lidocaína 5\%) e vasoconstritor (oximetazolina 0,05\%) em uma fossa nasal, com nasofibroscópio flexível de 3,2 mm da marca Mashida ${ }^{\circledR}$, acoplado à microcâmera da marca Asap ${ }^{\circledR}$ e documentado em DVD. Neste exame, além de estudar o tamanho e a relação da tonsila faríngea na nasofaringe, era avaliada a posição do septo nasal, tamanho dos cornetos, polo superior das tonsilas palatinas, presença de secreção e outras lesões em fossa nasal e hipofaringe.

Para a determinação do grau de hiperplasia de tonsilas faríngeas na nasofibrofaringoscopia, foi utilizada a classificação na qual é avaliada a relação das tonsilas faríngeas com outras estruturas presentes na nasofaringe (15):

- Grau 1-tonsila sem contato com estruturas da nasofaringe (Figura 1);

- Grau 2 - tonsila em contato com tórus tubário (Figura 2);

- Grau 3 - tonsila em contato com tórus tubário e vômer (Figura 3);
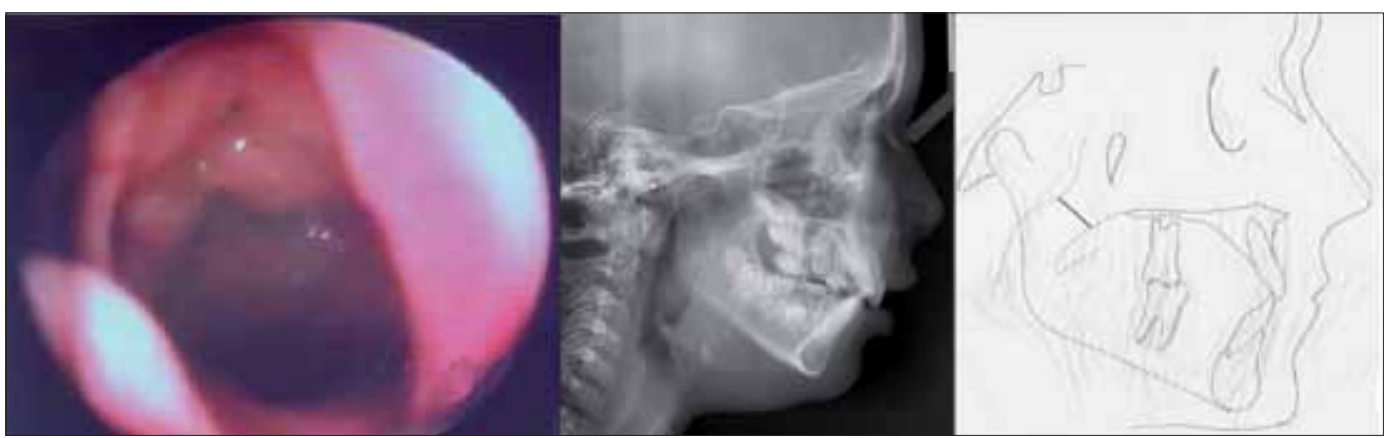

Figura 1. Hiperplasia das tonsilas faríngeas Grau 1 na nasofibrofaringoscopia e cefalometria (telerradiografia e traçado cefalométrico)
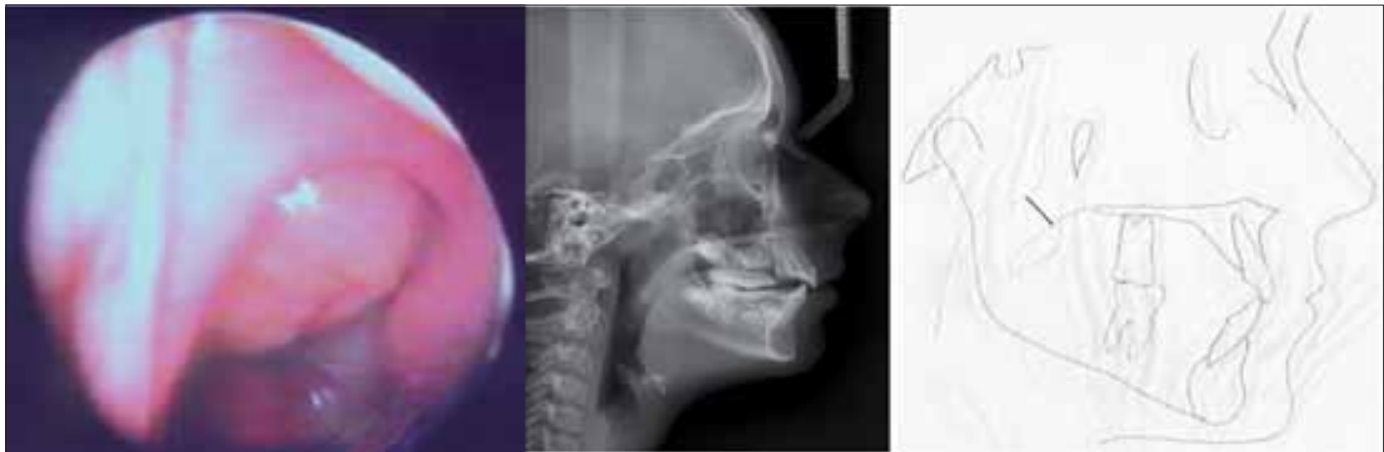

Figura 2. Hiperplasia das tonsilas faríngeas Grau 2 na nasofibrofaringoscopia e cefalometria (telerradiografia e traçado cefalométrico) 


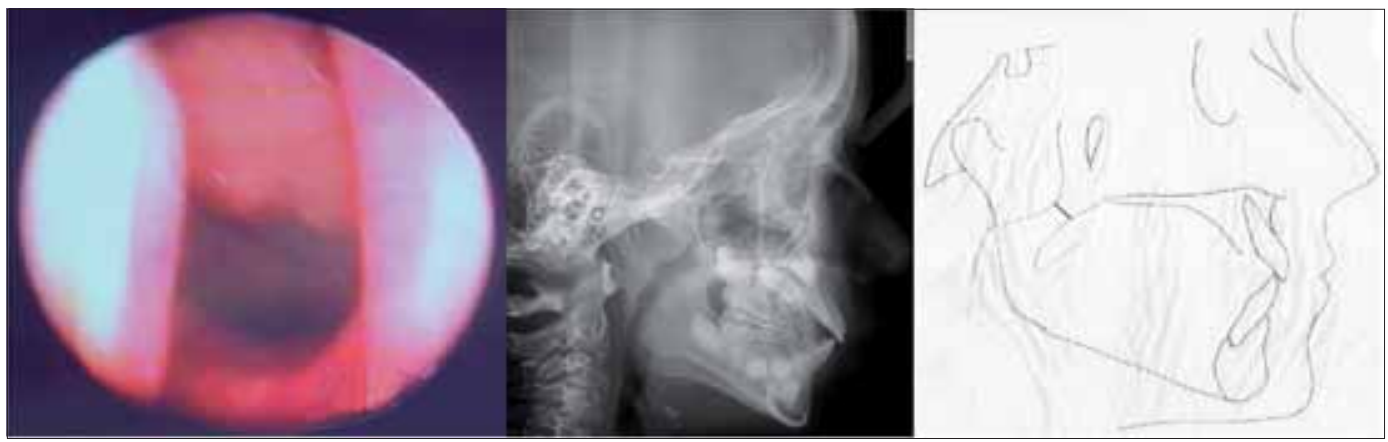

Figura 3. Hiperplasia das tonsilas faríngeas Grau 3 na nasofibrofaringoscopia e cefalometria (telerradiografia e traçado cefalométrico)

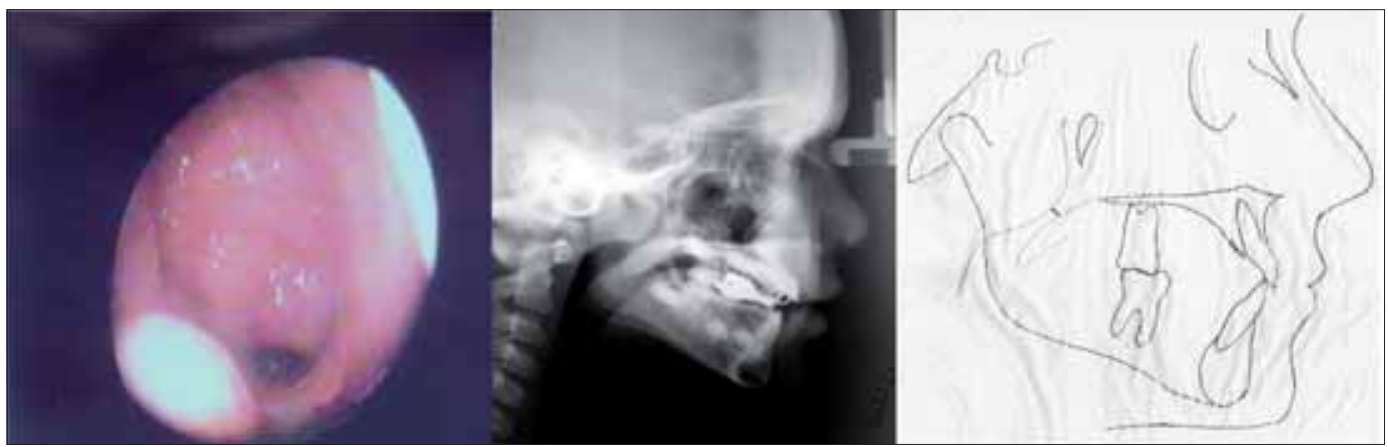

Figura 4. Hiperplasia das tonsilas faríngeas Grau 4 na nasofibrofaringoscopia e cefalometria (telerradiografia e traçado cefalométrico)

- Grau 4 - tonsila em contato com tórus tubário, vômer e palato mole em repouso (Figura 4).

Posteriormente as crianças foram encaminhadas para avaliação cefalométrica em serviço de radiologia odontológica, na qual foi obtida telerradiografia de perfil esquerdo do crânio com uso de cefalostato para o perfeito posicionamento do paciente a uma distância de $1,5 \mathrm{~m}$. Na radiografia obtida em norma lateral foi realizado traçado cefalométrico computadorizado através do software Sistema cef X - Sistema de cefalometria computadorizada versão 2.4.0.0 da CDT software - consultoria, desenvolvimento e treinamento de informática. O exame foi realizado sempre com o mesmo aparelho e avaliado pelo mesmo profissional especialista em radiologia odontológica.

Para determinação do grau de hiperplasia das tonsilas faríngeas na análise cefalométrica, foi usada a classificação na qual é mensurado em milímetros o espaço aéreo entre o bordo faríngeo do palato e o ponto mais próximo da faringe (16):

- Grau 1 - espaço nasofaríngeo maior que 6 mm(Figura 1);

- Grau 2 - espaço nasofaríngeo entre 4,1 e 6 mm(Figura 2);
- Grau 3 - espaço nasofaríngeo entre 2,1 e 4 mm (Figura 3);

- Grau 4 - espaço nasofaríngeo entre 0 e 2 mm (Figura 4).

Para verificar a correlação entre os exames de nasofibrofaringoscopia e cefalometria, utilizou-se o teste de correlação para postos de Spearman ao nível de significância de $5 \%$. Os testes de correlação podem resultar em um número entre -1 e +1 , sendo que o zero significa que não há correlação, o -1 que há correlação perfeita negativa e o +1 que existe correlação positiva perfeita; quanto mais próximo de +1 , maior a correlação entre as variáveis testadas. Os possíveis resultados desse teste para uma correlação positiva são (17):

- igual a 0 quando não há correlação;

- entre 0 e 0,3 para uma correlação fraca;

- entre 0,3 e 0,6 para uma correlação regular;

- entre 0,6 e 0,9 para uma correlação forte;

- entre 0,9 e 1 para uma correlação muito forte;

- igual a 1 quando há uma correlação perfeita dos dados.

Para calcular a sensibilidade, especificidade, valor preditivo positivo e valor preditivo negativo do exame 
radiológico, a nasofibrofaringoscopia foi considerada o exame de referência (padrão ouro) e foi necessário estabelecer valores limites para determinar se os exames eram positivos ou negativos. Assim, os exames nasofobrofaringoscópico e cefalométrico graus I e II foram considerados negativos e os exames graus III e IV foram considerados positivos.

\section{RESULTADOS}

Na Tabela 1 é apresentado o resultado da correlação entre os exames de nasofibrofaringoscopia e cefalometria no diagnóstico do grau de hiperplasia de tonsilas faríngeas.

A partir da análise dos resultados da Tabela 1, verifica-se coeficiente de correlação Rho de Spearman igual a 0,52. Este resultado evidencia associação regular e positiva entre os exames de nasofibrofaringoscopia e cefalometria $(\mathrm{p}=0,000)$ ao nível de significância de 5\%.

Na Tabela 2 é apresentada a distribuição dos resultados dos exames de nasofibrofaringoscopia e cefalometria utilizados para avaliação de testes diagnósticos.

Na Tabela 2, considerandoa nasofibrofarinsgoscopia como padrão-ouro para o diagnóstico da hiperplasia de tonsilas faríngeas, verificou-se que a cefalometria teve sensibilidade (proporção entre o número de diagnósticos cefalométricos radiográficos positivos corretos e o número total de casos positivos) de 35\%. A especificidade da cefalometria (proporção entre o número de diagnósticos negativos corretos e o número total de casos negativos) foi de $97 \%$. O valor preditivo positivo do exame cefalométrico (proporção entre o número de diagnósticos cefalométrico positivos corretos e o número total de diagnósticos cefalométricos positivos) foi de $87 \%$. O valor preditivo negativo (proporção entre o número de diagnósticos cefalométricos negativos corretos e o número total de diagnósticos cefalométricos negativos) foi de $72 \%$.

\section{DISCUSSÃO}

A hiperplasia de tonsilas faríngeas é uma das principais causas de obstrução do trato respiratório superior. Por essa razão, estudos têm sido desenvolvidos com o objetivo de avaliar a confiança dos procedimentos diagnósticos para detecção da obstrução da nasofaringe em decorrência do aumento de tonsilas faríngeas. Os exames mais comumente usados são radiografia de cavum, nasofibrofaringoscopia flexível e rígida, podendo ser utilizado também a cefalometria $(11,13,18)$.

Observa-se na literatura uma preocupação quantoà melhor forma de diagnosticar e tratar crianças com suspeita
Tabela I. Correlação entre os exames de nasofibrofaringoscopia e cefalometria no diagnóstico do grau de hipertrofia de tonsila faríngea

\begin{tabular}{lccc}
\hline $\begin{array}{l}\text { Exame } \\
\text { Obstrução }\end{array}$ & $\begin{array}{c}\text { Nasofibro- } \\
\text { faringoscopia }\end{array}$ & Cefalometria & \\
\hline Grau I & $n=15$ & $n=32$ & \\
& $27,27 \%$ & $58,18 \%$ & \\
Grau2 & $n=20$ & $n=15$ & \\
& $36,36 \%$ & $27,27 \%$ & $R s=0,52$ \\
Grau3 & $n=19$ & $n=5$ & $p=0,000$ \\
& $34,54 \%$ & $9,09 \%$ & \\
Grau4 & $n=1$ & $n=3$ & \\
& $1,83 \%$ & $5,46 \%$ & \\
\hline
\end{tabular}

n=número de crianças; $p=$ nível de significância de 5\%; $\mathrm{Rs}=$ coeficiente de correlação Rho de Spearman.

Tabela 2. Distribuição das crianças conforme resultados dos exames de nasofibrofaringoscopia e cefalometria na avaliação de testes diagnósticos.

\begin{tabular}{lccc}
\hline Cefalometria & \multicolumn{2}{c}{ Nasofibrofaringoscopia } & Total \\
& Positivo & Negativo & \\
\hline Positivo & 7 & I & 8 \\
Negativo & 13 & 34 & 47 \\
\hline Total & 20 & 35 & 55 \\
\hline
\end{tabular}

de hiperplasia de tonsilas faríngeas, situação frequente na prática otorrinolaringológica $(13,19)$. Em vista disso, este estudo teve o intuito de correlacionar os exames de nasofibrofaringoscopia e cefalometria no diagnóstico da hiperplasia de tonsilas faríngeas, destacando-se as principais contribuições dos mesmos.

Através dos achados desta pesquisa verificou-se uma correlação regular e positiva, Rho de Sperman de 0,52, salientando-se que não foi encontrada uma correlação perfeita entre os dois exames. Foi constatado que a maioria das crianças apresentou hiperplasia de tonsilas faríngeas graus 2 e 3 , seguidas de grau 1 na nasofibrofaringoscopia e a maior parte das crianças apresentou hiperplasia de tonsilas faríngeas grau 1 , seguidas de grau 2 na avaliação cefalométrica (Tabela 1). A partir da análise desses resultados, pode-se inferir que a cefalometria apresentou tendência a subestimar o tamanho da tonsila faríngea na nasofaringe em relação à nasofibrofaringoscopia.

A sensibilidade do exame cefalométrico, ou seja, a probabilidade de dar resultado positivo quando o paciente apresenta a condição, foi de $35 \%$. O valor preditivo negativo, que expressa a probabilidade de o indivíduo não apresentar o problema quando o resultado do exame é negativo, foi de $72 \%$. Esses resultados sugerem que exame 
cefalométrico negativo pode não estar avaliando corretamente a nasofaringe quando comparado com exame nasofibrofaringoscópico.

Resultados semelhantes também foram observados em outro estudo semelhante (13), que compararam radiografia de cavum e nasofibrofaringoscopia flexível para avaliar o grau de obstrução da nasofaringe. Os autores concluíram que crianças com sintomas clássicos de obstrução respiratória, na ausência de hipertrofia de tonsilas faríngeas no exame radiológico, devem ser submetidas à nasofibrofaringoscopia flexível para maior acurácia diagnóstica.

Vários estudos considerama nasofibrofaringoscopia como exame padrão ouro para avaliação da nasofaringe $(12,13,20-22)$. Isso é justificado, entre outros motivos, pela visão dinâmica e tridimensional da nasofaringe, que possibilita analisar não só o tamanho da tonsila faríngea, mas a sua relação com as demais estruturas dessa região. Isso pode explicar por que ao comparar-se a nasofibrofaringoscopia com a cefalometria, o segundo exame tende a subestimara relação das tonsilas faríngeas na nasofaringe.

$\mathrm{Na}$ prática clínica muitas vezes verifica-se que pacientes com sintomas de respiração oral que apresentam no exame radiológico tamanho da tonsila faríngea normal ou próximo do normal, ao serem avaliados pela nasofibrofaringoscopia podem a presentar hiperplasia de tonsilas faríngeas bem como outras alterações, tais como hipertrofia cauda corneto inferior e desvio de septo posterior.

Essa afirmação é reforçada por outro estudo (11), no qual foram avaliadas através de nasofibrofaringoscopia 45 crianças entre quatro e 12 anos, todas apresentando obstrução nasal crônica por hiperplasia de tonsilas faríngeas e radiografia de cavum sem alterações. Os resultados obtidos mostraram a presença de 27\% (17 casos) de tonsilas faríngeas consideradas grandes, $42 \%$ (24 casos) de tonsilas faríngeas de tamanho moderado e 31\% (19 casos) de tonsilas faríngeas pequenas. Os exames detectaram também a presença de oito casos de hipertrofia de cauda de cornetos inferiores (13,3\%) e quatro casos de desvio septal posterior (6,6\%). Esses a chados reforçam a importância da indicação da nasofibrofaringoscopia em crianças com obstrução nasal e com exame radiológico normal, por permitir uma avaliação direta, tridimensional e dinâmica da área do cavum.

Por outro lado, outro estudo (23) que verificou a eficácia da radiografia cefalométrica lateral no diagnóstico da hiperplasia de tonsilas faríngeas através da comparação com a endoscopia nasal, difere dos resultados desta pesquisa, pois os autores evidenciaram que a radiografia cefalométrica lateral se mostrou um exame eficiente por apresentar sensibilidade, especificidade e valores preditivos e negativos altos para o diagnóstico de hiperplasia de tonsilas faríngeas. Concluíram que apesar da superioridade da nasofibrofaringoscopia em avaliar a nasofaringe, o exame de cefalometria negativo para hipertrofia de tonsila faríngea seria suficiente para excluir esta patologia.

Outro estudo evidenciou através das informações de três unidades vinculadas ao sistema único de saúde (SUS), que a respiração oral é a queixa otorrinolaringológica mais frequente e que a radiografia de cavum foi o exame mais solicitado pelos otorrinolaringologistas (19). A partir dos resultados do presente estudo, acredita-se que se for considerado apenas a radiografia na detecção da hiperplasia de tonsilas faríngeas, o diagnóstico e o tratamento adequado do respirador oral podem ficar comprometidos, uma vez que em alguns casos o grau de obstrução da tonsila faríngea foi subestimado em relação ao exame nasofibrofaringoscópico.

A respiração oral na infância quando não tratada acarreta alterações craniofaciais e dentárias; alteração dos órgãos fonoarticulatórios; alterações corporais; alterações nas funções orais e outras alterações como do sono, nutricionais, do comportamento, entre outras (24). Todas essas alterações evidenciadas no respirador oral necessitam de uma intervenção multiprofissional que incluem médicos, fonoaudiólogos, odontólogos, fisioterapeutas, entre outros (1).

Embora a cefalometria correlaciona-se apenas de maneira regular com a nasofibrofaringoscopia no diagnóstico do respirador oral, ela pode complementar o exame endoscópico fornecendo informações sobre o crescimento facial, permitindo a documentação deste e seu monitoramento. Essas informações serão úteis para os demais profissionais envolvidos no atendimento do paciente com respiração oral.

A cefalometria identifica muitas alterações presentes no respirador oral de maneira precoce e fornece informações sobre a nasofaringe para o otorrinolaringologista, alterações morfológicas do sistema estomatognático para o fonoaudiólogo e sobre crescimento facial e oclusão dental para o odontólogo $(3,14,23)$. Assim, a cefalometria colabora não só para o diagnóstico, mas também para reduzir custos e integrar os diversos profissionais da equipe multidisciplinar envolvida no atendimento do respirador oral.

Uma pesquisa que comparou (25) a radiografia de cavum com a cefalometria, encontrou no primeiro exame 61\% de má posicionamento do paciente. Já na cefalometria é utilizado o cefalostato para controlar o posicionamento do paciente o que explica a superioridade deste exame em relação à radiografia de cavum. 
Embora a nasofibrofaringoscopia seja o exame padrão ouro para avaliação da nasofaringe, a cefalometria é um exame que o complementa fornecendo informações sobre crescimento craniofacial e possibilitando documentação e monitoramento do tratamento das complexas alterações morfofuncionais relacionadas à respiração oral.

Tendo em vista que neste estudo a cefalometria apresentou tendência a subestimar o tamanho da tonsila faríngea em relação à nasofibrofaringoscopia, sugere-se que os pacientes que apresentam sintomatologia de respiração oral e radiografia normal, sejam encaminhados para avaliação complementar com a nasofibrofaringoscopia, que possibilita maior precisão diagnóstica por ser um exame tridimensional e dinâmico.

\section{CONCLUSÃO}

A avaliação da nasofaringe de crianças pode ser feita pela nasofibrofaringoscopia e pela cefalometria, pois esses exames apresentam uma correlação regular e positiva. No entanto, a partir da análise dos resultados deste estudo, verificou-se que a cefalometria tende a subestimar o tamanho da tonsila faríngea em relação à nasofibrofaringoscopia. Assim, na presença de cefalometria negativa para obstrução na nasofaringe em paciente com sintomas de respiração oral, sugere-se a realização da nasofibrofaringoscopia.

\section{REFERÊNCIAS BIBLIOGRÁFICAS}

1. Weckx LLM, Weckx 1Y.Respirador bucal: causas e conseqüências. Rev Bras de Medicina. 1995, 52(8):86374.

2. Lessa FCR, Enoki C, Feres MFN, Valera FCP, Lima WTA, Matsumoto MAN. Influência do padrão respiratório na morfologia craniofacial. Rev Bras Otorrinolarigol. 2005, 71(2):156-60.

3. Mocellin M, Fugmann EA, Gavazzoni FB, Ataide AL, Ouriques FL, HerreroJr. F. Estudo cefalométrico-radiológico e otorrinolaringológico correlacionando o grau de obstrução nasal e o padrão de crescimento facial em pacientes não tratados ortodonticamente. Rev Bras de Otorrinolarigol. 2000, 66(2):116-20.

4. Motonaga SM, Berte LC, Anselmo-Lima WT. Respiração bucal: Causas e alterações no sistema estomatognático. Rev Bras de Otorrinolarigol. 2000, 66(4):373-79.

5. Cuccia AM, Lotti M, Caradonna D. Oral breathing and head posture. Angle Orthodontist. 2008, 78(1):77-82.
6. Harari D, Redlich M, Miri S, Hamud T, Gross M. The effect of mouth breathing versus nasal breathing on dentofacial and craniofacial development in orthodontic patients. Laryngoscope. 2010, 120(10):2089-93.

7. Junqueira P. Respiração oral: considerações fonoaudiológicas. In: Junqueira P; Dauden AT. Aspectos Atuais em Terapia Fonoaudiológica II. São Paulo: Pancast; 2002, p.32-42.

8. Junqueira P. Avaliação miofuncional. In: Marchesan IQ. Fundamentos em Fonoaudiologia - Aspectos Clínicos da Motricidade Oral. $2^{\underline{a}}$ ed. Rio de Janeiro: Guanabara-Koogan; 2005, p.19-28.

9. Wang DY, Bernheim N, Kaufman L, Clement P. Assessment of adenoid size in children by fibreoptic examination. Clin Otolaryngol Allied Sci. 1997, 22(2):1727.

10.Monteiro ECM, Pilon RR, Dall`Oglio G, Giovanna P. Estudo da hipertrofia adenoidiana: endoscopia X radiografia de nasofaringe. Rev Bras Otorrinolaringol. 2000, 66(1):912.

11.Souza BB, Hennemann GV, Anselmo-Lima WT. Importance of nasal fiberotic examination in the presence of a normal x-ray of cavum. Int J Pediatr Otorhinolaryngol. 2000, 55(1):29-32.

12. Mlynarek A, Tewfik MA, Hagr A, Manoukian JJ, Schloss MD, Tewfik TL et al. Lateral Neck Radiography versus Direct Video Rhinoscopy in Assessing Adenoid Size.J Otolaryngol. 2004, 33(6):360-5.

13.Lourenço EA, Lopes KC, Pontes Júnior A, Oliveira MH, Umemura A, Vargas AL. Estudo comparativo radiológico e nasofibroscópico do volume adenoideano em crianças respiradoras orais. Rev Bras Otorrinolaringol. 2005, 71(1):238.

14. Bianchini AP. A cefalometria nas alterações miofuncionais orais diagnóstico e tratamento fonoaudiológico. São Paulo: Pró-Fono; 2002.

15.Parikh SR, Coronel M, Lee JJ, Brown SM. Validation of a new grading system for endoscopic examination of adenoid hypertrophy. Otolaryngol Head Neck Surg. 2006, 135(5):684-7.

16. McNamara Jr JA.A method of cephalometric evaluation. Am J Orthod. 1984, 86(6):449-69.

17.Callegari-Jacques SM. Bioestatística: princípios e aplicações.Porto Alegre: Artmed; 2003. 
18. Chisholm EJ, Lew-GorS, Hajioff D, Caulfield H.Adenoid size assessment: a comparison of palpation, nasendoscopy and mirror examination. Clin Otolaryngol. 2005, 30(1):3941.

19.T-Ping C, Weckx LLM. Atendimento otorrinolaringológico do Sistema Único de Saúde de crianças e adolescentes em três municípios brasileiros. Rev Bras de Otorrinolarigol. 2008, 74(4):571-8.

20. Wormald PJ, Prescott CA. Adenoids:comparison of radiological assessment methods with clinical and endoscopic findings. J Laryngol Otol. 1992, 106(4):342-4.

21. Finkelstein Y, WexlerD, Berger G, Nachmany A, ShapiroFeinberg M, Ophir D. Anatomical basis of sleep-related breathing abnormalities in children with nasal obstruction. Arch Otolaryngol Head Neck Surg. 2000, 126(5):593-600.
22. Abdollahi-Fakhim S, Naderpoor M, Shahid N, Javadrashid R, Mashrab O, Ravaghi M. Assessment of adenoid size in children. Res J Biol Sci. 2008, 3(7):747-9.

23.Castellucci-Barbosa M, Knop LAH, Lessa MM, Araujo TM. Avaliação da radiografia cefalométrica lateral como meio de diagnóstico da hipertrofia de adenóide. R Dental Press Ortodon e Ortop Facial. 2009,14(4): 83-91.

24. Marchesan IQ, Krakauer LR. The importance of respiratory activity in myofunctional therapy. Int J Orofacial Myology. 1996, 22:23-7.

25. Ikino CMY, D‘Antonio WEPA, de la Cortina RAC, Lessa M, Castilho AM, Goto EY, Butugan O, Amaral TS. Teleradiologia lateral de crânio e radiografia de cavum: Estudo comparativo em crianças com obstrução nasal. Rev Bras Otorrinolaringol. 2000, 66(6):592-6. 